\title{
Téoros
}

Revue de recherche en tourisme

\section{Un fleuve inacessible}

\section{Stéphane Bouchard et Léonce Naud}

Volume 20, numéro 1, printemps 2001

Mers et littoraux

URI : https://id.erudit.org/iderudit/1071904ar

DOI : https://doi.org/10.7202/1071904ar

Aller au sommaire du numéro

Éditeur(s)

Université du Québec à Montréal

ISSN

0712-8657 (imprimé)

1923-2705 (numérique)

Découvrir la revue

Citer cet article

Bouchard, S. \& Naud, L. (2001). Un fleuve inacessible. Téoros, 20(1), 9-12. https://doi.org/10.7202/1071904ar d'utilisation que vous pouvez consulter en ligne.

https://apropos.erudit.org/fr/usagers/politique-dutilisation/ 


\title{
Un fleuve inacessible
}

\author{
* Retourne sur tes pas, 0 ma vie, tu vois bien que la rue est fermé... * \\ Anne Hebert, Tombeau des rois
}

\section{Stéphane Bouchard ef \\ Léonce Naud}

\begin{abstract}
Si vous n'êtes pas vous-mêne une embarcation, vous aurez des problèmes à accéder aux plans d'eau du Québec. Depuis une génération, des centaines de millions de dollars en fonds publics ont servi à faciliter la délicate mise à l'ean et le remisage dans des parkings liquides de yachts de plusieurs tonnes. Cependant, $i l$ existe fort peu d'endroits où une jeune mère peut faire patauger son rejeton dans l'eau pour le rafraichir, même s'il a chaud et ne pèse que

quelques kilos.
\end{abstract}

D epuis trois décennies, les Quebecois ont consacré plusieurs milliards de dollars à l'assainissement des eaux. Ces efforts portent maintenant fruit : la qualité de l'eau du fleuve Saint-Laurent et de nos grands cours d'eau navigables s'est grandement améliorée. Il est ici question de l'eau dans laquelle on se baigne, on pêche ; de l'eau sur laquelle on fait du canot, du kayak, du dériveur, de la voile; de l'eau que l'on côtoie en marchant sur la greve ; bref, de l'eau du fleuve.

Cependant, la pratique d'activités aquatiques en été et sur glace en hiver présupposent des accès publics bien aménagés aux plans d'eau. On pourrait penser que le Québec étant faiblement peuplé, cette question ne se pose pas, mais ce serait oublier que les deux tiers des Québécois (4,3 millions de personnes) habitent désormais à l'intérieur d'une zone de dix kilomètres de largeur de chaque côté du fleuve. Il est probable que nous assisterons dans les prochaines décennies à un renforcement de cette tendance. $\mathrm{Or}_{\text {, }}$ en raison d'une occupation privative des rives, de larges sections du littoral fluvial sont totalement dépourvues d'accès publics, au moment même où la population s" attend à tirer profit des efforts consentis à dépolluer les eaux au Québec. Une telle situation résulte, d'une part, de carences en matière de droit québécois de l'eau et, d"autre part, de pratiques qui transforment graduellement le fleuve en canal inutilisable pour la plupart des usages publics ou privés (par des enrochements tous azimuts, entre autres).

\section{Le droit québécois de I'eau}

Contrairement à ce qui prévaut dans la plupart des pays occidentaux, le Québec ne dispose d'aucune législation expresse quant à l'accès public aux rives et aux littoraux. Les cours d'eau navigables el flottables font pourtant partie du domaine public et à cette propriété publique correspond, en principe, un acces public. En effet, 1 article 920 du Code civil du Québec prevoit que a toute personne peut circuler sur les cours d'eau et les lacs . Mais le probleme de l'acces a ces cours d'eau demeure entier, puisque ce même article soumet ce droit à d'importantes restrictions : a à condition de pouvoir y accéder légalement, de ne pas porter atteinte aux droits des propriétaires riverains, de ne pas prendre pied sur les berges et de respecter les conditions de l'utilisation de l'eau $\$$. Dans les nombreux endroits où l'on trouve une occupation privative intense des rives. ces restrictions sont suffisantes pour priver la très grande majorité de la population et les touristes cventuels - de l'usage d'un bien qui appartient bel et bien au domaine public.

Au Québec, le droit d'acces à l'eau est un attribut de la propriété. La propriété privée étant le droit de priver les autres de l'usage d'un bien que l'on possede, un propriétaire riverain n'est donc aucunement tenu de tolérer que l'on passe sur sa propriété pour accéder à un cours d'eau, même si le cours d'eau est public. Par ailleurs, en milieu urbain, les acces publics relèvent des municipalités, ce qui n'est pas toujours un facteur d'encouragement au caractère accessible des rives et des littoraux. 
Pourtant, il y a déjâ eu dans l"histoire du droit québécois des mesures législatives garantissant le caractere public des rives. L'ordonnance De la Marime (1681), laquelle déclarait non aedificandi le littoral lui-même, avait elle-même été précédée en Nouvelle-France d"une mesure législative concemant le fleuve Saint-Laurent, adoptée par le Conseil souverain, à savoir * [...] qu'il reste deux perches libres audessus des plus hautes marées, pour la liberté tant du passage des charrettes et bestiaux que de la navigation s. Ces deux perches de servitude publique équivalaient à 36 pieds $(11,6$ mètres $)$.

Une fois la Nouvelle-France réduite à la Province of Quebec (1763), le droit littoral dans ladite Province s'alignera progressivement sur les intérêts commerciaux du XIX siècle industriel, c'est-âdire principalement sur le commerce du bois et les activités portuaires. L'ancienne servitude de 36 pieds a éte abolie en 1850 par une loi stipulant qu" « aucune personne n'entrera ni ne passera [...] le long d'aucune rivière ou ruisseau [...] sans la permission du propriétaire [...] » (Brun, 1969 : 23). En 1857, le législateur précisera la notion d'utilisation publique des cours d'eau et de leur's rives : cela signifie a la ravigation et le transport du bois * (lbid. : 29).

Dans le passé, le droit québécois de l'eaı a donc dejà comporté des dispositions qui garantissaient le caractere public des rives, dispositions quil ont cependant été abolies ill y a un siècle et demi, sous la pression d'intérêts privés. L'état actuel de la législation québecoise, moins soucieuse des droits publics que ne l'était le droit littoral français d'avant 1760 , apparaît encore plus choquant quand on se livre à un exercice de droit comparatif.

En France, le législateur a jugé nécessaire, à partir des années soixante, de renforcer le caractère public des plans d'eau et des accès à ces derniers. C'est ainsi que les piétons disposent d'un droit de passage paisible sur une bande de trois mètres sur l'ensemble des propriétés situées sur le bord de mer. Cependant, c'est une chose de pouvoir circuler le long du rivage, cela en est une autre de pouvoir y accéder. En maints endroits, une occupation privative du bord de mer rendrait inopérant ce droit. Conséquemment, la Loi du 3 janvier 1986 pernet l'ouverture de passages transversaux pour accéder au rivage à partir d'une voie publique et ce, a tous les 500 metres; c'est 1'État qui détermine et aménage à ses frais cette servitude de passage. En ce qui concerne les plages, la loi française repose sur le principe que * l'usage libre et gratuit par le public constitue la destination fondamentale des plages $\omega$.

Cette liberté d'accês par tous aux rivages et aux plages de France constitue sans aucun doute l"une des causes fondamentales de l'extraordinaire popularité de ce pays à titre de destination touristique $(74,5$ millions de visiteurs en 2000). Que deviendrait $I^{*}$ économie des régions littorales françaises si l'on introduisait en France le droit québecois de l'eau, selon lequel a le public n'a pas accès aux rives on tant que publicl ? Bien plus, au-delà même des voies d'acces, de nombreux pays européens ont promulgué des interdits de construction sur le littoral non encore urbanise. Cette zone littorale non aedificandi n' $\mathrm{n}$ 'st alors pas inférieure à 100 mètres ; il va sans dire que de telles interdictions n’existent pas au Québec.

\section{Aux États-Unis, la doctrine du "Public Trust"}

Plus près de nous, les Américains favorisent cux aussi l'accessibilite publique aux plans d'eau. Adopté en 1972, le Coastal Zone Management Act definit les principes que doivent appliquer les États en matière de gestion cottière. Ils doivent assurer $\alpha$ a public access to the coasts for recreation purposes $\%$. Pour ce faire, on invoque la doctrine du Public Trust ; prenant appui sur le code Justinien ${ }^{2}$, ce corpus législatif soutient que l'air, les cours d'eau ainsi que la mer-rivages inclus - sont communs à toute l'humanité. Dans eette optique, les gouvernements ne sont que les gestionnaires des ressources riveraines dont la propriété revient de plein droit aux citoyens : tout l'espace intertidal en plus d'une partie de la plage au-dessus font ainsi automatiquement partie du bien commun.

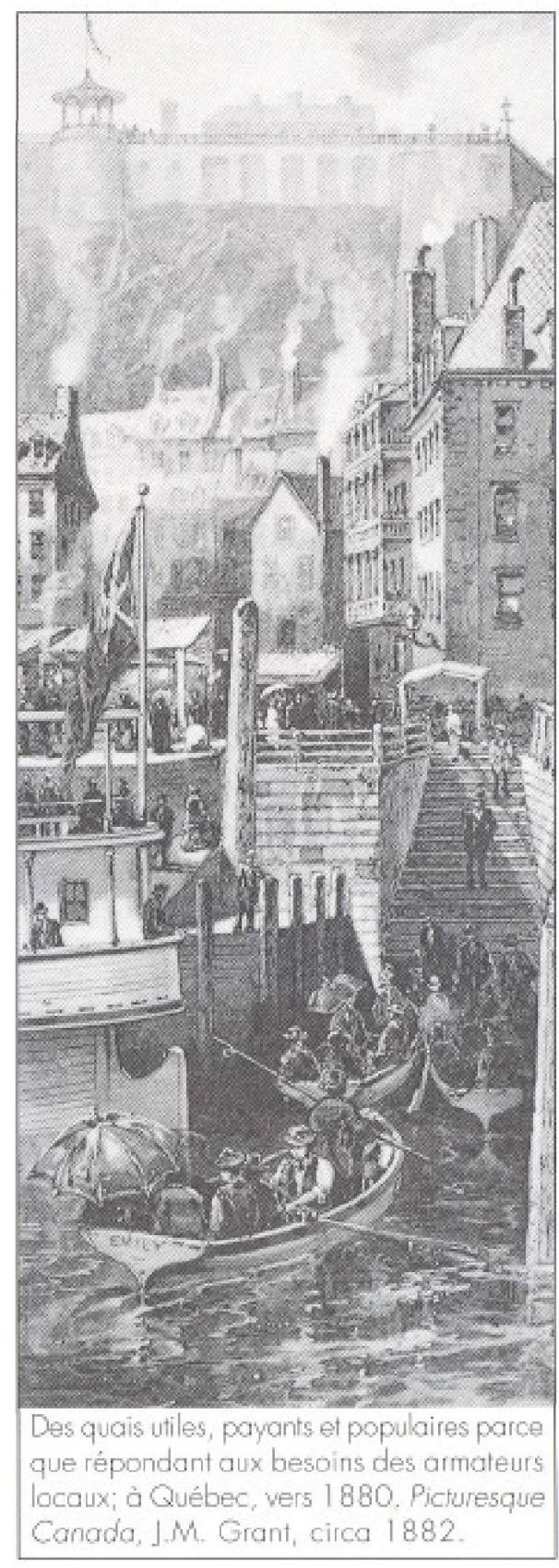

\section{Inaccessibilité également physique}

Favoriser - si necessaire avec des fonds publics - l'acces et l'usage des littoraux, des fleuves, des rivières, des lacs et des autres plans d'eau par l'ensemble de la population d'un pays constitue l'un des fondements les plus sûrs de progrès économique et social. En plus des ports militaires et commerciaux ou des marinas, ouvrages côtiers à accès contrôlé, réserves en pratique aux travaux ou a la jouissance de membres de corporations privées, un État soucieux du bien public mettra en 
place des structures riveraines d'accueil destinces à des usages accessibles au grand public et d'intérêt pour ce dernier.

L'utilisation des plans d'eau pour le commerce, la récréation, le tourisme et le loisir repose géographiquement sur de rares points d'ancrage dispersés ici et là le long des rives, essentiellement des quais, des estacades, des bassins et d'autres ouvrages publics. Il convient donc d'en rếpartir sagement l'accès et I'usage entre plusieurs catégories concurrentes d'utilisateurs, tant pour les générations actuelles que futures.

Plusieurs ont déjà signalé qu'à l'urbanisation graduelle des littoraux fluviaux et maritimes correspond, en pratique, la disparition d'une multitude de voies d'accès aux cours d'eau - la plupart informelles - dont ont depuis toujours bénéficié les populations humaines et animales. En de nombreux pays, les fleuves sont en voie de devenir, comme jamais auparavant, fermés aux accès et aux usages généraux.

Par exemple, au cour des villes, là où vit maintenant la majorité de la population,

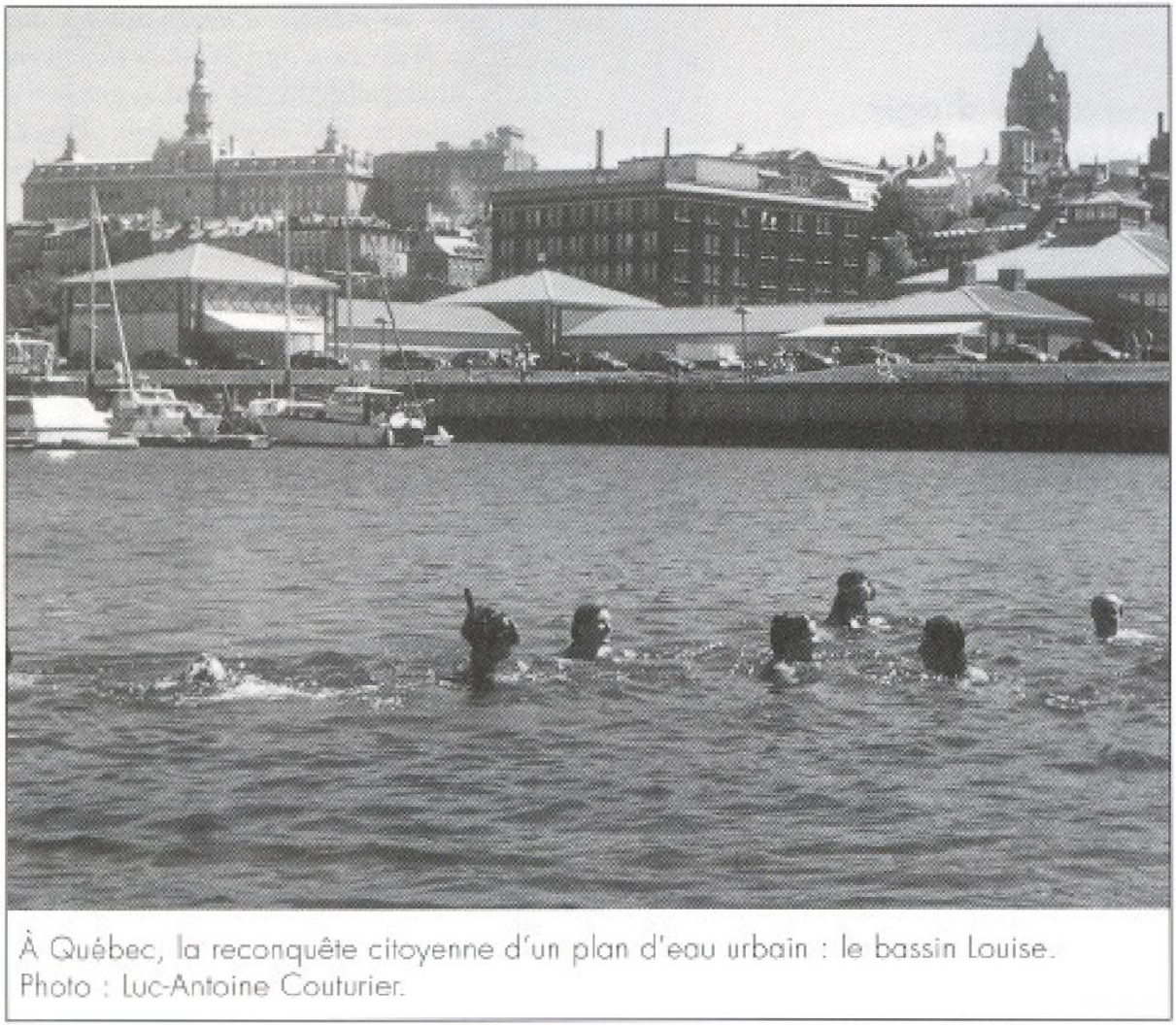

il convient de récuser avec force cette conception réductrice selon laquelle un accès à l'eau se résume à un trottoir ou à un quai sur lequel le public ne peut que déambuler, parfois littéralement coincé entre un parc de stationnement ou un édifice et une surface liquide en contrebas, cette dernière inaccessible pour quelque usage que ce soit. De tels « déambulatoires tristes $*$ seraient d'ailleurs inappropriés même pour des populations animales utilisatrices d'un point d'eau, car la surface liquide elle-même demeure interdite d'usage (Prelorenzo, 1993).

Dans le cas de plans d'eau urbains, 1'accès public à l'eau signifie, entre autres, la présence en plusieurs endroits d'escaliers, de gradins, de surfaces et de plans inclinés, de pentes qui permettent aux gens d'atteindre l'eau, d'y toucher, voire d'y entrer et d'en ressortir ; bref, de l'utiliser de diverses façons. La plupart des usages publics nécessitent l'utilisation de terrains riverains assez vastes adjacents aux plans d'eau. C'est pourquoi l'érection de bâtiments privés sur les rivages urbains est généralement perçue comme contraire à l'intếrêt public. À l'avenir, les progrès
A l'aube du nouveau millénaire, rades et bassins fluviaux en milieu urbain trouveront leur rentabilité en offrant une polyvalence de services. Jeux aquatiques, baignade, pêche récréative, location de voiliers, forfaits de pêche, bateaux d'excursion et de plaisance, tous solliciteront l'usage de ces plans d'eau protégés. Rives artificielles, digues, jetées et promenades devront être conçues expressément pour en favoriser l'accès et l'usage par le grand public en toutes saisons.

\section{Le fleuve: un grand canal enroché ?}

À ce sujet, il faut souligner l'urgence de s'interroger publiquement sur certaines pratiques actuelles. Au cours des dernières années, pour réduire les coûts d'entretien, la plupart des quais du Saint-Laurent se sont vus corsetés dans de prodigieux amoncellements de roches. Gilbert Normand, secrétaire d'État au gouvernement fédéral canadien, a ainsi déploré « qu'entre Gaspé et Lévis, on ne peut plus accrocher une chaloupe nulle part ${ }^{3} \%$. L'opération ne 
laisse souvent subsister qu' une plate-forme de béton dépourvue de toute fonction, de tout attrait ou de toute utilité. Nous n'avons alors plus affaire à des quais, mais à de gros tas de roches avec une plaque de béton au milieu, dont les municipalités prennent alors livraison. Ces tas de roches représentent de véritables no man's land où devient impossible tout usage humain ou animal de cette fragile zone d'interface entre la terre ferme et un plan d'eau, à l'exception de certains types de pêche à la ligne. De nombreux brise-lames construits à la vavite ces dernières années présentent des caractéristiques analogues. Il faut ajouter à cela plusieurs dizaines de kilomètres de rives fluviales et littorales d'ores et déjà dûment enrochées... pour l'éternité ?

Comment les enfants de demain pourront= ils aller jouer sur les grèves du fleuve comme les générations précédentes ont pu le faire, si les rives sont enrochées, souvent aux endroits précis où elles seraient les plus accessibles au grand public ? Comment pourra-t-on aller se baigner, pêcher au milieu de gros bloes dont la seule et unique fonction consiste à briser la force des vagues ? Comment un passant pourrat-il s'approcher de l'eau, y toucher sans danger? Comment pourra-t-on amarrer une chaloupe sans crainte de la voir mise en pièces par le premier coup de vent qui pourrait l'amener à donner contre les rochers? Comment les arbres arriverontils à pousser sur la rive, ombrageant l'eau de leur feuillage?

Déjà, dans l'Empire romain, selon I'historien Pline le Jeune, les ingénieurs construisaient des brise-lames « par des travaux dignes d'être contemples : un grand navire transporte de gros rochers ; ceux-ci sont immergés, entassés et retenus par leur seul poids, et l'ensemble monte en une sorte de digue $[\ldots] \%$. Cependant, Pline ajoute que, par la suite, \& l'on couronnait avec d'autre matériel cet entassement de blocs de pierre, de sorte que l'ensemble prenne peu à peu l'aspect d'une île naturelle ${ }^{4} \%$. On pratiquait done dejà, sous Trajan, l'aménagement paysager des enrochements, ouvrages rébarbatifs à tous les usages ou presque. Les ingénieurs romains trouvaient done important de leur donner un caractère plus naturel et par conséquent plus accueillant pour les multiples activités humaines.

\section{Conséquence économique des littoraux}

Un littoral accessible et convivial constitue un attrait touristique de premier ordre. Des études américaines ont montré que les plages sont l'elément clé du tourisme ; à titre d'exemple, les États côtiers reçoivent $85 \%$ de tous les revenus liés au tourisme aux États-Unis. En France, on a évalué à 100 millions de francs le préjudice causé par le déficit d'image affectant les côtes atlantiques françaises, en ráison des multiples articles de presse au sujet de la marée noire causée par le naufrage du pétrolier Érika (plus de 12 millions en Belgique, 15 millions aux Pays-Bas, 5 millions en Grande-Bretagne [Bostnavaron, 2000]). Au Québec, à-t-on déjầ réalisé une seule estimation de la valeur économique de nos littoraux maritimes ou fluviaux ou encore de l'ampleur du préjudice financier causé par certains types d'aménagements, par exemple la destruction, par enrochement ou autrement, de la plupart des plages publiques dont pouvait s"enorgueillir le Saint-Laurent il y a une cinquantaine d'années?

\section{L'urgence d'agir}

Le Québec accuse un retard considérable en matière de protection des aceès publics au littoral. Pourtant, il est important de s'assurer que les generations futures pourront jouir elles aussi de ce bien inestimable. Un littoral accessible constitue au surplus un attrait touristique d'importance. Il est essentiel d'adopter des mesures législatives qui permettront de préserver les accès publics existants sur l'ensemble du littoral et de désenclaver ce dernier là où les accès font actuellement défaut.

Ainsi, nous proposons qu'un groupe de travail dresse un portrait clair de l'accessibilité passée et présente aux rives du Saint-Laurent, aux cours d'eau navigables, aux lacs, aux réservoirs et aux autres plans d'eau ; qu'il examine les législations étrangères en matière d'accès public à l'eau et de préservation du littoral et qu'il définisse le mandat d'un organisme analogue au
Conservatoire du littoral français. Cette unité aurait pour vocation unique la préservation et la mise en valeur des rivages et des littoraux québecois dans le respect des interêts du plus grand nombre. Les Québécois n' habiteront leur territoire que lorsqu'ils y auront véritablement un accès libre et entier. La conservation du caractère public des rives est un enjeu national.

Słéphane Bouchard, diplômé en philosophie de l'Université de Montréal. est conseiller au ministère du Conseil exécutif, gouvernement du Québec. Il auve egalement au sein de groupes de citoyens qui veulent redonner aux Québécois l'accès à leurs plans d'eau et l'usage de ceux-ci.

Léonee Naud, diplômé en philosophie et en géographie de I'Université d'Ottawa, s'interesse aux relations urbano-portuaires ainsi qu'au devenir des rivages urbains. Il collabore avec plusieurs réseaux internationaux, dont l'Association internationale Villes et Ports (Le Havre) et le Centro Internazionale Cintà d'Acqua (Venise).

\section{Notes}

1 wotre seconde question a trait aux legislations en matiere d'access publics anx rives. Il $n$ "y en a pas. Selon ce document [Le droit québécois de l'eau], le public n'a pas d'accès aux rives; le droit d'accès appartiendrait aux riverains et il n'y a pas de loi là-dessus. o (Latulippe, 1999).

2 Corpus Juris Civilis, Ve siecle apres J.-C.

3 In Le Soleil (Québec), 17 août 1998.

4 Lettre à Comelianus, ler siêcle apress J.-C.

\section{Bibliographie}

Bostnavaron, François (2000), \& La saison touristique estivale s" annonce aussi bonne que celle de 1999 \%, Le Monde, Paris, éd. électronique, 16 aoụt.

Brun, Henri (1969), Histoire du droit quebecois de l'eau 1663-1969, Gouvemement du Québec.

Latulippe, Jean-Maurice (1999), Direction des politiques du secteur municipal, Ministère de l'Environnement du Québec. Correspondance, 14 mai.

Prelorenzo, Claude (1993), La ville au bord de l'eau, Paris, Parenthèses. 